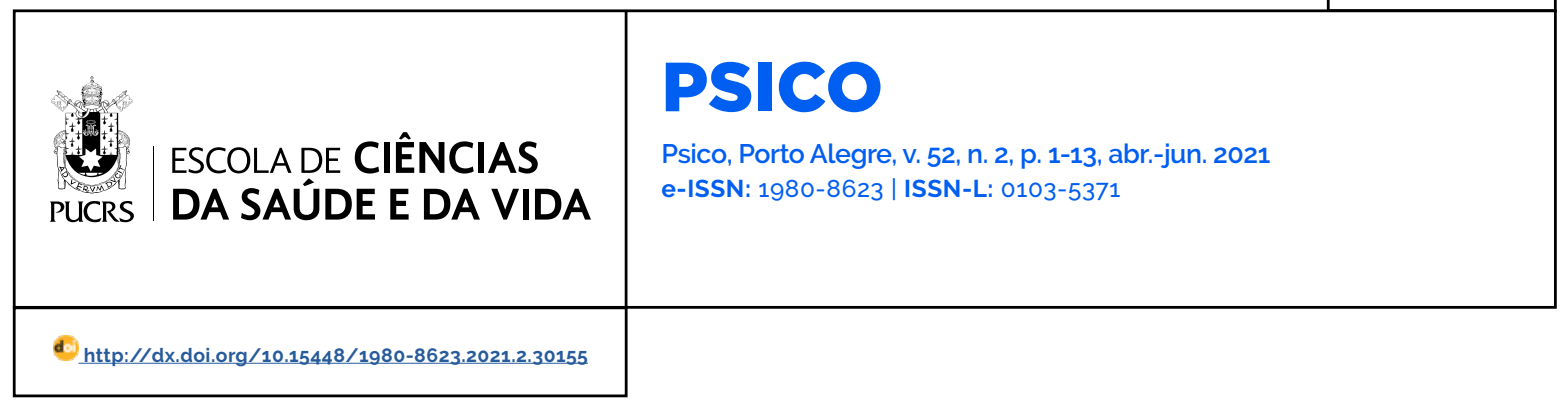

\title{
Estresse, desesperança e expectativas de futuro na adolescência em alunos do ensino médio
}

\author{
Stress, hopelessness and future expectations in adolescence in student of the high school \\ El estrés, la desesperanza y las expectativas futuras en la adolescência en estudiantes \\ de escuela secondaria
}

\section{Cybelle Bezerra Sousa Florêncio ${ }^{1}$}

orcid.org/0000-0002-9392-7109 cybelle.flor2@gmail.com

\section{Edson Marcos Leal \\ Soares Ramos ${ }^{1}$ \\ orcid.org/0000-0001-5425-8531 \\ ramosedson@gmail.com}

\section{Simone Souza da Costa Silva ${ }^{1}$}

orcid.org/0000-0003-0795-2998

symon.ufpa@gmail.com

Recebido em: 19 mar. 2018. Aprovado em: 4 mar. 2021. Publicado em: 10 ago. 2021
Resumo: O presente artigo investigou a associação dos niveis de estresse, desesperança e expectativas de futuro em uma amostra de 295 adolescentes, alunos do ensino médio de uma rede educacional particular confessional (sendo 127 meninos (43,1\%) e 168 meninas (56.9\%); com idades entre 14 e 18 anos ( $M=15,90 ; S D=1,21$ ), na região metropolitana de Belém, no estado Pará. Os instrumentos utilizados foram: questionário sociodemográfico, Escala de Stress para Adolescentes (ESA), Escala $\mathrm{BECK}$ de Desesperança (BHS) e questionário sobre expectativas futuras. Por meio de técnicas estatísticas para análise dos dados, observou-se que as meninas estavam mais estressadas que os meninos e que adolescentes com moderado estresse apresentaram alto nivel de desesperança. Também se verificou que adolescentes com altos niveis de estresse apresentaram baixa ou muito baixa expectativa de ser respeitado na comunidade e concluir o ensino médio. Esse estudo sugere que o estresse pode comprometer as expectativas de futuro e o bem-estar do adolescente. Palavras-chave: adolescente, estresse, desesperança, expectativas

Abstract: This paper investigated the association levels of stress, hopelessness and future expectations in a sample of 295 teenagers, high school students of a private confessional education network (with 127 boys (43.1\%) and 168 girls (56.9 $\%)$ ); $14-18$ years $(M=15.90 ; S D=1.21$ ), in the metropolitan region of Belém, Pará. The instruments used were: demographic questionnaire, Stress Scale for Adolescents (ESA), Beck Hopelessness Scale (BHS) and questionnaire about future expectations. Through statistical techniques for data analysis, it was observed that the girls were more stressed than children and adolescents with moderate stress had high levels of hopelessness. Also found that adolescents with high levels of stress have low or very low expectation of being respected in the community. This study suggests that stress can compromise the future of expectations and well-being of adolescents. Keywords: adolescent, stress, hopeless, expectations

Resumen: El presente artículo investigó la asociación de los niveles de estrés, desesperanza y expectativas de futuro en una muestra de 295 adolescentes, alumnos de la Enseñanza Media de una red educativa particular confesional (siendo 127 niños (43,1\%) y 168 niñas $(56,9 \%$ ) con edades entre 14 y 18 años ( $M=$ $15,90, S D=1,21$ ), en la región metropolitana de Belém, en el estado del Pará. Los instrumentos utilizados fueron: cuestionario socio demográfico, Escala de Estrés para Adolescentes (ESA), Escala BECK de Desesperanza (BHS) y cuestionario sobre expectativas futuras. Mediante técnicas estadisticas para el análisis de datos, se observó que las niñas estaban más estresadas que los niños y adolescentes con moderado estrés presentaron un alto nivel de desesperanza, también se verificó que adolescentes con altos niveles los niveles de estrés presentaron baja o muy baja expectativa de ser respetados en la comunidad y concluir la Enseñanza Media. Este estudio sugiere que el estrés puede comprometer las expectativas de futuro y el bienestar de los adolescentes cente.

Palabras clave: adolescente, estrés, desesperanza, expectativas 
No mundo globalizado, a lógica contemporânea tem afetado as concepções atuais sobre o trabalho, a família, as relações. As mudanças socioeconômicas muito rápidas, especialmente nos últimos 20 anos, geraram beneficios e problemas à vida humana (Khan, 2013), promovendo o desequilibrio entre as capacidades da pessoa e as demandas do ambiente (Marques et al., 2015). Ao olhar para o passado, sugere-se que a humanidade avançou alcançando uma vida melhor. No entanto os custos emocionais também parecem maiores agora do que antes, especialmente, para adolescentes que ainda estão construindo sua identidade (Khan, 2013).

A adolescência, uma etapa da vida caracterizada por várias transformações de ordem biológica, psicológica e social e com uma tendência a ser naturalizada como um periodo problemático e turbulento tem recebido uma atenção especial nas últimas décadas por pesquisadores do desenvolvimento humano (Steinberg \& Morris, 2001; Santrock, 2014).

A despeito do crescente interesse nos últimos anos dos pesquisadores pela adolescência, no Brasil há uma carência de serviços públicos que contribuam à promoção da saúde mental, emocional e o bem-estar de crianças e de adolescentes (Rocha et al., 2013). Com uma população de 34.257.621 individuos entre 10 e 19 anos, de acordo com o Censo Demográfico (Instituto Brasileiro de Geografia e Estatística [IBGE], 2010), é relevante saber como os adolescentes brasileiros estão vivendo as transições em suas vidas na contemporaneidade urbana e o que esperam do futuro quando consideram seu presente, a fim de contribuir com a construção de políticas públicas compativeis com suas reais necessidades.

$\mathrm{Na}$ adolescência o individuo já apresenta a capacidade de projetar metas para o futuro baseado em suas expectativas. Diante dos desafios de adaptá-las as exigências da vida contemporânea e as mudanças que ocorrem nesse periodo da existência, problemas psicológicos podem advir (Caires \& Silva, 2011). A World Health Organization (WHO, 2012) afirma que alguns transtornos mentais podem ter início na infância e pré-adolescência, tais como o transtorno de ansiedade e a depressão, esta última com prevalência alta em populações adolescentes (Caspi et al., 2014; Khan, 2013; Patias et al., 2016).

É importante ressaltar que as próprias mudanças biopsicossociais e, especialmente, as hormonais, presentes no período da adolescência, tornam os adolescentes bem mais sensiveis a eventos estressores ecológicos, sendo essa uma das explicações apresentadas por estudiosos para o aumento dos episódios depressivos nesta fase da vida (Caires \& Silva, 2011; Schraml et al., 2011; Landstedt \& Gadin, 2012). Rocha et al., (2013) afirmam que essas mudanças naturais da adolescência frente a eventos estressores aumentam a chance de episódios depressivos de cinco para 20\%. Outros autores revelam que niveis elevados de estresse podem evoluir para transtornos que levem o individuo até mesmo ao suicídio (WHO, 2012; Patias et al., 2016), sendo essa a segunda maior causa de morte na população adolescente (Thapar et al., 2012).

O estresse pode comprometer forças psicológicas, como a esperança, do individuo que precisam ser acionadas para enfrentar as dificuldades. Folkman (2010) afirma que para enfrentar altos niveis de estresse, em um tempo prolongado, a esperança é uma ferramenta essencial para as pessoas embora não seja uma forma permanente, podendo estar ausente, indisponivel em alguns momentos na vida. Quando a esperança está ausente, então há a desesperança que é caracterizada por pensamentos autoderrotistas, uma visão pessimista, negativa ou nula frente ao futuro, e está intensamente relacionada à depressão (Beck et al., 1997).

Em um estudo com 134 adolescentes euro-americanos sobre estresse, esperança e solidão, Yarcheski et al. (2011) relatou uma correlação moderada e inversa entre estresse e esperança, e uma correlação positiva entre estresse e solidão. Chou et al. (2011) examinaram as relações entre o estresse, estratégias de enfrentamento, e sintomas depressivos em 756 estudantes universitários chineses em Taiwan. Os resultados do estudo sugerem que altos niveis de estresse estão associado a sintomas depressivos e, também, com as estratégias de enfrentamento. 
A esperança é uma vantagem significativa sobre o estresse e tem relação com o enfrentamento, segundo Folkman (2010). A autora ainda sugere que é a esperança que tem o papel de sustentare promover o enfrentamento. A esperança consiste no processo de pensamento a respeito dos objetivos de um individuo seguido da motivação para atuar nesse sentido. De acordo com a Teoria da Esperança, ela é a energia cognitiva que pode ser usada para estabelecer objetivos e traçar estratégias para o alcance das mesmas (Snyder, 1995), colaborando com o desenvolvimento positivo.

As perspectivas futuras têm sido associadas com o comportamento no presente, assim como desempenham um papel importante no desenvolvimento positivo do jovem (Callina et al., 2014). Um estudo realizado por Burrow et al. (2010) com 318 adolescentes entre 14 e 18 anos, investigou o papel do propósito (objetivo ou projeto) na vida do jovem utilizando a Escala de Propósito para Jovens, de Bundick et al. (2006), e a Escala da Esperança de Snyder et al. (1991). Os dados revelaram que a afinidade entre exploração e compromisso com o projeto de vida tem implicações importantes no desenvolvimento da identidade e do bem-estar do adolescente, levando-o à capacidade de engajamento.

Considerando que a adolescência é um periodo que parece propor mudanças efetivas no individuo que podem desencadear quadros expressivos de estresse e de desesperança, é importante verificar se tais fatores podem influenciar nas expectativas de futuro do adolescente (Bock, 2004). Assim, há uma problemática para ser discutida: é possivel que o estresse possa alterar o nivel de desesperança do adolescente e impactar suas expectativas de futuro? Deste modo, o presente estudo tem por objetivo verificar a associação de expectativas de futuro com os indices de estresse e de desesperança de adolescentes que não se encontram em situação de vulnerabilidade social.

\section{Método}

Este estudo foi organizado em um formato quantitativo, transversal, descritivo e correlacional, com o objetivo de descrever os níveis de estresse e de desesperança em adolescentes escolares da região metropolitana de Belém, estado do Pará, procurando uma associação entre essas variáveis e sua influência sobre as expectativas de futuro. A pesquisa ocorreu no periodo de fevereiro a junho de 2015.

\section{Participantes}

A presente pesquisa contou com a participação de 295 adolescentes, alunos do ensino médio de uma rede particular e confessional de ensino. Essa amostra foi calculada tomando como referência a população de 651 estudantes das três séries do ensino médio nas três escolas particulares envolvidas na pesquisa, com técnica de amostragem probabilistica aleatória.

A amostra foi composta por 295 alunos do ensino médio, sendo 120 da $1 .^{a}$ série (40,68\%), 90 alunos da 2. ${ }^{\text {a }}$ série $(30,51 \%)$ e 85 alunos da $3 .^{\text {a }}$ série (28,81\%). Com idades entre 14 e 18 anos ( $M=15,90$ anos; SD = 1,21), a saber: 14 anos (14,58\%), 15 anos (24,75\%), 16 anos (27,11\%), 17 anos (23,05\%) e 18 anos (10,51\%). 127 adolescentes eram do sexo masculino (43,05\%) e 168 do sexo feminino (56,95\%). Todos os participantes têm residência fixa, a saber: 61,70\% moram com os pais, $23,05 \%$ moram com o pai ou a mãe, 9,15\% moram com os avós, e os demais $(6,1 \%)$ vivem com outros parentes ou adultos não familiares.

Para a participação na pesquisa foi exigido o Termos de Consentimento Livre e Esclarecido (TCLE), devidamente assinado pelo participante e/ou seu responsável, em caso de o participante ser menor de 18 anos. Não foi exigido pelo Comitê de Ética de Medicina Tropical, à época da aprovação do projeto de pesquisa, o termo de assentimento do participante, por isso esse documento não consta como parte do procedimento legal. Sendo assim, foram excluídos da amostra participantes que não apresentaram até a data da aplicação dos instrumentos na unidade de ensino, o Termo de Consentimento Livre e Esclarecido devidamente assinado.

\section{Ambiente}

A pesquisa foi realizada em três unidades escolares com ensino médio, de uma rede confessional estabelecidas na região metropolitana de Belém, Pará. 


\section{Instrumentos}

Questionário Sociodemográfico. Elaborado pelos pesquisadores para obtenção de informações pessoais tais como: sexo, idade, naturalidade, escolaridade.

Questionário sobre expectativas futuras. Organizado por Dell'Aglio et al. (2011), e apresentado no Questionário da Juventude Brasileira, na questão 76, é uma versão reduzida da Escala de expectativas quanto ao futuro, adaptada por Günther e Günther (1998). Esse instrumento teve por objetivo avaliar nove expectativas de futuro, a saber: (a) Concluir o Ensino Médio; (b) Entrar na Universidade; (c) Ter um emprego que me garanta boa qualidade de vida; (d) Ter minha casa própria; (e) Ter um trabalho que me dará satisfação; (f) Ter uma familia; (g) Ser saudável a maior parte do tempo; (h) Ser respeitado na minha comunidade; e (i) Ter amigos que me darão apoio. Esses itens foram avaliados dentro de uma escala Likert de cinco pontos, a saber: 1 (muito baixas); 2 (baixas); 3 (cerca de 50\%); 4 (altas); e 5 (muito altas) - Tabela 2.

Escala de Estresse para Adolescentes - ESA (Tricolli \& Lipp, 2011). Instrumento de avaliação psicológica aprovado pelo Conselho Federal de Psicologia no Brasil, em 2005, que permite verificar a existência ou não de estresse nos adolescentes pesquisados. É uma escala composta por 44 itens que objetiva identificar a frequência e a intensidade em que os sintomas do estresse aparecem a partir das reações em quatro dominios, a saber: psicológicas, cognitivas fisiológicas e interpessoais. Consta de uma escala do tipo Likert de cinco pontos, que revela a experimentação das reações do estresse apontadas pelos itens. No estudo de Schermann et al. (2014) com uma comunidade adolescente, realizado nas cidades de Campo Grande, MS, João Pessoa, PB, e São Paulo, SP, a ESA apresentou propriedades psicométrica apropriadas com coeficiente de alfa de Cronbach de 0,9394 para frequência (sintomas) e 0,9398 para intensidade (fase).

Escala Beck de Desesperança - BHS (Cunha, 2011). Criada por Aaron T. Beck e colaboradores, foi adaptada no Brasil por Jurema Alcides Cunha (2011). É um instrumento estruturado de autoadministração composto por afirmativas que medem, a partir das atitudes negativas referentes ao futuro, o seu grau de pessimismo e, consequentemente, a desesperança. É uma escala dicotômica (certo e errado) e seus itens são pontuados em o ou 1, com intervalo de pontuação de 0-20, onde a soma de seus itens individuais resulta em um escore total, e a partir desse escore dá-se a classificação da desesperança. Esse instrumento foi validado no Brasil com a seguinte classificação: zero a 4-mínimo; 5 a 8-leve; 9 a 13- moderado e 14 a 20- grave (Cunha, 2001). No estudo de Langaro e Benetti (2014), a BHS obteve dados psicométricos aceitáveis a partir do coeficiente de consistência interna de 0.81 .

\section{Procedimento}

\section{Procedimentos éticos}

O projeto desta pesquisa foi submetido ao Comitê de Ética de Medicina Tropical da Universidade Federal do Pará, conforme resolução 466/12 do Conselho Nacional de Saúde, sob o número CAAE 40645414.0.000.5172 e aprovado pelo parecer de n. ${ }^{\circ}$ 1.069.887. A coleta de dados foi iniciada somente após a emissão do parecer de aprovação do Comitê e autorização das instituições participantes.

Os participantes e seus pais foram esclarecidos quanto ao sigilo das informações e de identidade, objetivos, riscos e benefícios da pesquisa e só então foi assinado o Termo de Consentimento Livre e Esclarecido pelo participante e pelo responsável legal, quando este era menor de idade.

\section{Procedimentos de coleta de dados}

A pesquisa foi dividida em 4 etapas, a saber:

1. Contato com as instituições (três unidades de ensino médio da rede educacional) e solicitação de autorização para realização da pesquisa.

2. Seleção dos participantes (visita às salas de aulas para entrega das cartas explicativas com os objetivos da pesquisa e os Termos de Consentimento - TCLE - para serem assinados).

3. Conferência dos TCLE e marcação da data para aplicação dos instrumentos nas salas de aula. 
4. Coleta de dados (aplicação dos instrumentos nas salas de aula). Os instrumentos foram aplicados aos participantes em um período de aula que corresponde a 45 minutos.

\section{Análise de dados}

Após a realização de todas as etapas da coleta de dados os testes foram corrigidos e os dados catalogados e lançados no programa Statistical Package for the Social Scienses - SPSS 24.0. A análise dos dados foi dividida em duas etapas com a utilização das técnicas estatísticas de Análise Descritiva (AD), para obtenção de medidas de sintese (quantidades, percentuais, médias, desvios-padrão), Análise Fatorial (AF), para a construção dos índices de desesperança de Beck e de estresse para adolescentes, Análise de Correspondência (ANCOR), para verificar a relação entre as categorias das variáveis do estudo e a Análise de Variâncias (ANOVA), para identificar diferenças entre as médias das variáveis em análise.

A primeira etapa foi iniciada com a análise descritiva da população sobre as variáveis sociodemográficas e as respostas ao questionário de expectativas de futuro buscando o registro das frequências e percentis destas variáveis (Tabelas 1 e 2).

Em seguida, foi utilizada a análise fatorial (AF), que é uma técnica estatística multivariada de independência, cuja função neste estudo foi encontrar fatores comuns em um conjunto de variáveis inter-relacionadas (Fávero et al., 2009). A partir da Análise Fatorial foi possível observar um considerável número de correlações significativas, com valores do nivel descritivo ( $p$-valor) inferiores a 0,05 (5\%) para as variáveis (perguntas) utilizadas na construção dos índices de estresse para Adolescentes e de desesperança de Beck, indicando que todas as variáveis (perguntas) são adequadas à aplicação da técnica de Análise Fatorial.

Tabela 1 - Estatísticas Resultantes da Aplicação da Técnica de Análise Fatorial as Variáveis Necessárias à Construção do Índice de desesperança do adolescente

\begin{tabular}{|c|c|c|c|c|c|c|c|}
\hline Variável & KMO & EsfericidadeBartlett & $\%$ Var. & MAA & Comum. & Correlação( $r)$ & EscoresFatoriais \\
\hline BHS1 & \multirow{20}{*}{0,828} & \multirow{10}{*}{$\chi^{2}=1177,51$} & \multirow{20}{*}{69,01} & $0,812^{\mathrm{a}}$ & 0,450 & $-0,482$ & $-0,096$ \\
\hline $\mathrm{BHS} 2$ & & & & $0,804^{a}$ & 0,127 & 0,341 & 0,060 \\
\hline $\mathrm{BHS}_{3}$ & & & & $0,812^{\mathrm{a}}$ & 0,705 & 0,786 & 0,780 \\
\hline $\mathrm{BHS}_{4}$ & & & & $0,764^{a}$ & 0,954 & 0,963 & 0,719 \\
\hline $\mathrm{BHS}_{5}$ & & & & $0,586^{a}$ & 0,997 & 0,991 & 0,960 \\
\hline BHS6 & & & & $0,614^{\mathrm{a}}$ & 0,122 & 0,310 & 0,170 \\
\hline $\mathrm{BHS7}$ & & & & $0,807^{a}$ & 0,345 & 0,553 & 0,076 \\
\hline BHS8 & & & & $0,614^{a}$ & 0,984 & 0,968 & 1,127 \\
\hline BHS9 & & & & $0,841^{\mathrm{a}}$ & 0,394 & 0,582 & 0,150 \\
\hline $\mathrm{BHS} 10$ & & & & $0,827^{a}$ & 0,869 & 0,878 & 0,807 \\
\hline $\mathrm{BHS} 11$ & & \multirow{10}{*}{$p=0,000$} & & $0,836^{a}$ & 0.596 & 0,759 & 0,415 \\
\hline $\mathrm{BHS} 12$ & & & & $0,881^{a}$ & 0,415 & $-0,462$ & $-0,101$ \\
\hline $\mathrm{BHS} 13$ & & & & $0,720^{a}$ & 0,151 & 0,370 & 0,151 \\
\hline BHS14 & & & & $0,723^{a}$ & 0,993 & 0,979 & 1,109 \\
\hline $\mathrm{BHS} 15$ & & & & $0,843^{a}$ & 0,622 & 0,598 & 0,114 \\
\hline $\mathrm{BHS} 16$ & & & & $0,853^{a}$ & 0,436 & 0,621 & 0,268 \\
\hline $\mathrm{BHS} 17$ & & & & $0,845^{\mathrm{a}}$ & 0,371 & 0,482 & $-0,026$ \\
\hline $\mathrm{BHS} 18$ & & & & $0,856^{a}$ & 0,668 & 0,590 & 0,733 \\
\hline $\mathrm{BHS} 19$ & & & & $0,897^{a}$ & 0,394 & 0,439 & 0,147 \\
\hline $\mathrm{BHS} 2 \mathrm{O}$ & & & & $0,855^{a}$ & 0,478 & 0,662 & 0,149 \\
\hline
\end{tabular}

Nota: KMO-Estatística de Kaiser-Meyer-Olkin; $\chi^{2}$ - Valordo Qui-quadrado ; $p$-Nível Descritivo; \% Var. - \% Variância Explicada pelo Fator; MAA-Medidade Adequação da Amostra; Comum.- Comunalidade. 
$6 / 13 \quad$ Psico, Porto Alegre, v. 52, n. 2, p. 1-13, abr.-jun. $2021 \mid$ e-30155

Tabela 2 - Estatísticas Resultantes da Aplicação da Técnica de Análise Fatorial as Variáveis Necessárias à Construção do Índice de estresse do adolescente

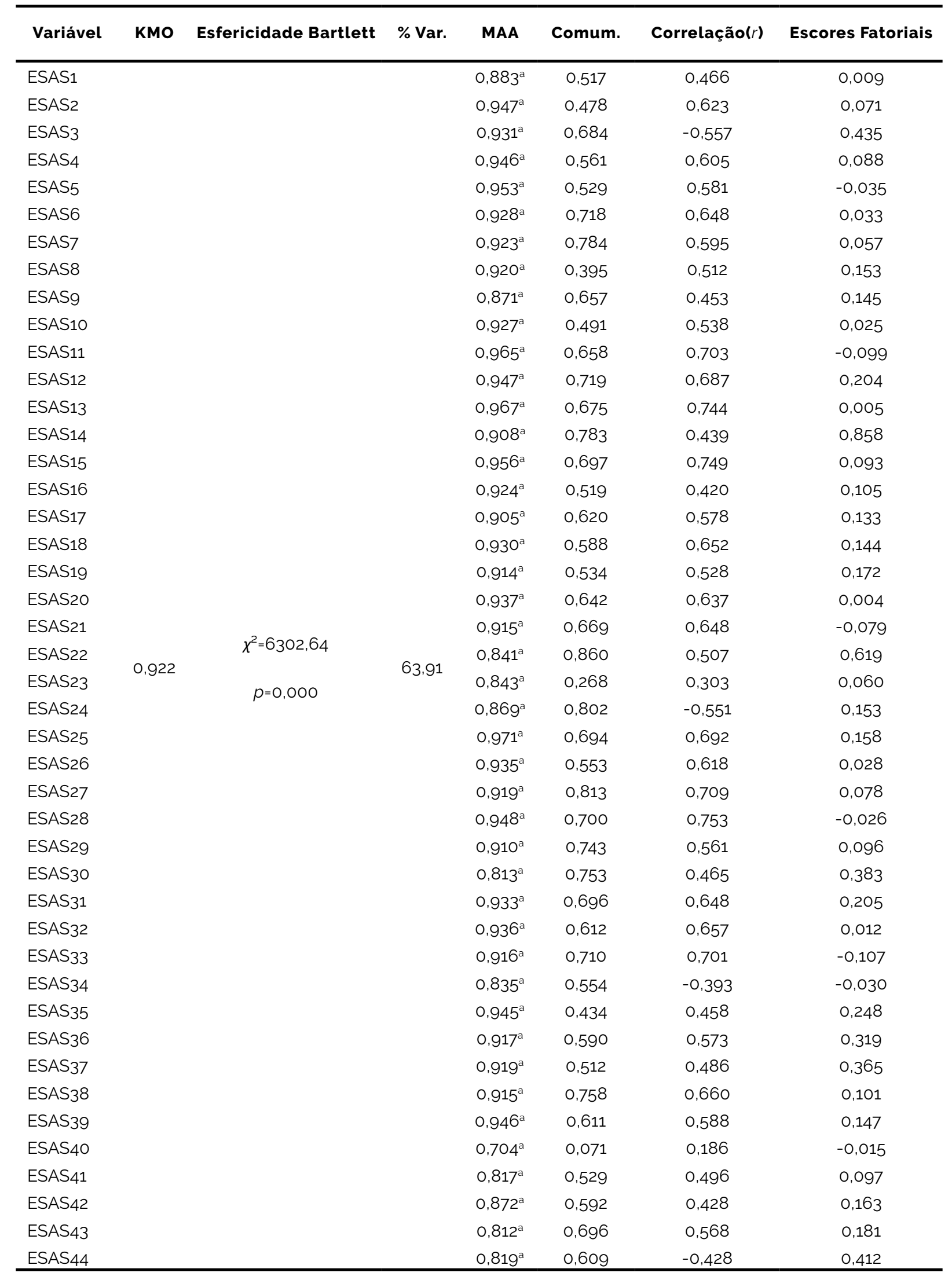

Nota: KMO - Estatística de Kaiser-Meyer-Olkin; $\chi^{2}$ - Valor do Qui-quadrado; $p$ - Nivel Descritivo; \% Var. - \% Variância Explicada pelo Fator; MAA-Medida de Adequação da Amostra; Comum. - Comunalidade. 
Os valores da estatística KMO na construção dos Índices de Desesperança de Beck (IDB) (KMO $=0,828)$ e Índice de Estresse para Adolescentes (IEA) (KMO = 0,922, foram superiores a 0,50, indicando que há adequação da Análise Fatorial ao conjunto de variáveis (perguntas). Além disso, o nivel descritivo do teste de esfericidade de Bartlett $(p=0,000)$ foi nulo, acarretando a rejeição da hipótese de a matriz de correlações ser a uma matriz identidade (Tabelas 1 e 2). Todos os valores do MAA para as variáveis (perguntas), necessárias à construção IDB e IEA, individualmente encontraram-se em domínio aceitável para a aplicação da técnica de Análise Fatorial, isto é, todos os valores de MAA foram superiores a 0,50 (Tabelas 1 e 2). Os fatores obtidos conseguirão restituir mais que $50 \%$ da informação do conjunto de variáveis (perguntas), como pode ser observado a partir do \% Var (Tabelas 1 e 2). Porém, o critério utilizado para retenção dos fatores não foi o \%Var restituido e sim o critério de Kaiser. Assim, um fator foi retido pelo critério de Kaiser, ou seja, aquele com autovalor superior a 1, para a construção do IDB e IEA (Tabelas 1 e 2). Além disso, as variáveis (perguntas) utilizadas na construção do IDB e IEA tiveram sua informação restituída de forma satisfatória pelo fator retido, já que apresentam maioria dos valores de comunalidade igual ou superior a 0,30 (30\%), isso foi possivel a partir do método rotacional Varimax (Tabelas 1 e 2). Finalmente, pode-se observar que as variáveis (perguntas) utilizadas na construção do IDB e IEA apresentaram em sua maioria no mínimo correlação moderada $(r \geq 0,50)$ com o fator (índice) obtido (Tabelas 1 e 2). Esses resultados respaldaram o emprego da Análise Fatorial para a extração de fatores e a estimação dos escores fatoriais e posterior construção do IDB e do IEA. Os valores positivos dos coeficientes das variáveis (perguntas), nas equações do IDB e do IEA, indicaram que quanto maior for o valor do escore obtido para um determinado adolescente, maior é o seu nivel de desesperança ou de estresse, respectivamente. Obtidos os escores fatoriais para BHS e ESA de cada adolescente foi realizada a padronização dos valores obtidos, para que os mesmos pudessem ser avaliados em uma escala de o a 1 ou o a $100 \%$.

A partir dos escores fatoriais obtidos por meio dos índices de desesperança de Beck (IDB) e de estresse para adolescentes (IEA) e com base na teoria dos quartis amostrais (Bussab \& Morettin, 2017), cada adolescente foi classificado em um dos seguintes três grupos distintos: de 0\% a 24,99\% (baixo estresse ou baixa desesperança), 25\% a 75\% (medianos níveis de estresse ou medianos níveis de desesperança) e de 75,01 a 100\% (maiores niveis de estresse ou maiores níveis de desesperança).

A segunda etapa da análise foi realizada com a técnica de Análise de Correspondência (ANCOR), que é uma técnica multivariada estatística (Fávero et al., 2009), a fim de verificar a dependência entre as categorias das variáveis em estudo (BHS, ESA, expectativas de futuro e dados sociodemográficos). Inicialmente, pode-se confirmar que os valores do nivel descritivo $(p)$ menores que o nível de significância de 0,05 (5\%) e do Critério Beta (B) maior que 3, indicam que tanto as variáveis em estudo como suas categorias são dependentes. Além disso, pode-se observar que a soma dos percentuais de inércia indica que mais de 70\% da informação foi restituida pela ANCOR. Desta forma, todos os pressupostos para utilização da técnica de Análise de Correspondência são satisfeitos (Ramos et al., 2008). A análise de correspondência foi realizada com o auxilio do aplicativo Statistica, versão 6.0. Em todos os testes, fixou-se $\alpha=5 \%(p \leq 0,05)$ para rejeição da hipótese nula.

\section{Resultados \\ Caracteristicas sociodemográficas}

Participaram desta pesquisa 295 estudantes do Ensino Médio, sendo 127 do sexo masculino e 168 do sexo feminino, com idades entre 14 e 18 anos. Na Tabela 3 é possivel encontrar informações relativas à análise descritiva e a percentuais relacionadas ao sexo, idade, série e moradia dos adolescentes participantes do estudo. 
Tabela 3 - Caracterização da Amostra

\begin{tabular}{lll}
\hline & Valor Bruto & Porcentagem \\
\hline Sexo & 127 & $43,05 \%$ \\
\hline Masculino & 168 & $56,95 \%$ \\
Feminino & & \\
Idade (M=15,90; SD 1,21) & & $14,58 \%$ \\
\hline 14 anos & 43 & $24,75 \%$ \\
15 anos & 73 & $27,11 \%$ \\
16 anos & 80 & $23,05 \%$ \\
17 anos & 68 & $10,51 \%$ \\
18 anos & 31 & \\
Série & & $40,68 \%$ \\
\hline $1^{\text {a Série }}$ & 120 & $30,51 \%$ \\
$2^{\text {a Série }}$ & $98,81 \%$ \\
$3^{\text {a Série }}$ & 90 & $61,70 \%$ \\
MORADIA & 85 & $23,05 \%$ \\
\hline Com os pais & & \\
Com o pai ou a mãe & 68 & \\
Com os avós & 27 & \\
rentes ou adultos & 182 & \\
\hline não-familiares & & \\
\hline
\end{tabular}

A média de idade dos adolescentes foi 15,90 anos (SD 1,21), a maior parte dos adolescentes (40,68\%) eram estudantes da $1{ }^{a}{ }^{a}$ série do ensino médio, moravam com os pais, e eram do sexo feminino. As raças foram representadas, a saber: branca (28,47\%); parda (60\%); negra (6,10\%); amarela (4,41\%); e indigena (1,02\%). Ressalta-se que $39,91 \%$ dos adolescentes declararam ser portadores de desabilidades, a saber: visual $(68,97 \%)$; auditiva (7,76\%); motora (7,76\%); intelectual (4,31\%), sendo que a maioria destes considerou sua desabilidade entre leve e moderada.

\section{Adolescentes e suas expectativas de futuro}

As expectativas de futuro foram levantadas a partir das respostas dos adolescentes ao Questionário sobre expectativas futuras, com escala Likert de 5 pontos a saber: 1 (muito baixas); 2 (baixas); 3 (cerca de 50\%); 4 (altas); e 5 (muito altas). Os dados apresentaram os resultados, que estão descritos na Tabela 4.

Tabela 4 - Expectativas de futuro dos adolescentes

\begin{tabular}{lccccc}
\hline $\begin{array}{l}\text { Variáveis das Expectativas de } \\
\text { Futuro em Porcentagens }\end{array}$ & Muito Baixas & Baixas & Cerca de $\mathbf{5 0 \%}$ & Altas & Muito Altas \\
\hline Concluir o Ensino Médio & 0,16 & 0,32 & 10,48 & 36,22 & $\mathbf{5 2 , 8 2}$ \\
Entrar na Universidade & 2,23 & 0,00 & 17,27 & 47,42 & 33,08 \\
Ter emprego & 0,08 & 0,83 & 15,50 & 44,00 & 39,59 \\
Ter casa própria & 0,97 & 0,00 & 12,09 & 37,39 & 49,55 \\
Trabalho e satisfação & 0,08 & 2,08 & 9,36 & 33,28 & $\mathbf{5 5 , 2 0}$ \\
Ter uma família & 0,49 & 1,96 & 13,70 & 25,12 & $\mathbf{5 8 , 7 3}$ \\
Ser saudável & 0,26 & 3,31 & 19,60 & 37,63 & 39,20 \\
Ser respeitado na comunidade & 0,25 & 1,32 & 13,11 & 39,57 & 45,75 \\
\hline
\end{tabular}

Os adolescentes apresentaram boas expectativas de futuro, destacando-se expectativas referentes a concluir o ensino médio (52,82\%), ter uma familia $(58,73 \%)$, trabalho e satisfação (55,20\%). Nota-se que concluir o ensino médio constitui uma expectativa de futuro a curto prazo, uma vez que se relaciona com ações alcançadas mais rapidamente; já as demais expectativas preveem sua realização em um tempo mais longo. 
Estresse $X$ Desesperança e as expectativas dos adolescentes

A partir da análise de correspondência (AC), foi verificada relação/associação entre as categorias das variáveis em estudo (BHS, ESA, expectativas de futuro e dados sociodemográficos). Algumas associações importantes foram verificadas e obtidas da AC que estão expostas na Tabela 5.

Tabela 5 - Residuo (Probabilidade) Resultante da Análise de Correspondência Aplicada ao Índice para Escala de Estresse para Adolescentes (ESA) e ao Índice para a Escala de Desesperança de Beck (BHS)

\section{ESA}

\begin{tabular}{|c|c|c|c|c|}
\hline & & Baixo & Moderado & Alto \\
\hline \multirow{3}{*}{$\mathrm{BHS}$} & Baixo & $-0,23(0,00)$ & $-0,59(0,00)$ & $1,06(71,04) *$ \\
\hline & Moderado & $0,31(24,28)$ & $-0,75(0,00)$ & $0,75(54,45)^{* *}$ \\
\hline & Alto & $-0,13(0,00)$ & $1,60(89,09)$ * & $-2,12(0,00)$ \\
\hline \multirow{2}{*}{ Sexo } & Masculino & $2,24(97,47)$ & $-0,11(0,00)$ & $-2,10(0,00)$ \\
\hline & Feminino & $-1,95(0,00)$ & $0,09(7,46)$ & $1,83(93,22)$ \\
\hline \multirow{3}{*}{ Série } & $1^{\mathrm{a}}$ & $2,62(99,13)$ & $-1,48(0,00)$ & $-0,57(0,00)$ \\
\hline & $2^{a}$ & $-1,23(0,00)$ & $0,82(58,65)$ & $0,09(7,11)$ \\
\hline & $3^{a}$ & $-1,85(0,00)$ & $0,91(63,96) * *$ & $0,58(43,81)$ \\
\hline \multirow{5}{*}{ Concluir o Ensino Médio } & Muito Baixas & $-0,71(0,00)$ & $-0,99(0,00)$ & $2,12(96,56)$ * \\
\hline & Baixas & $-0,71(0,00)$ & $-0,99(0,00)$ & $2,12(96,56) *$ \\
\hline & Cerca de $50 \%$ & $-1,85(0,00)$ & $1,12(73,70)$ * & $0,29(22,80)$ \\
\hline & Altas & $1,30(80,76) *$ & $-0,99(0,00)$ & $0,08(6,01)$ \\
\hline & Muito Altas & $0,03(2,56)$ & $0,51(39,33)$ & $-0,76(0,00)$ \\
\hline \multirow{5}{*}{$\begin{array}{l}\text { Ser } \\
\text { Respeitado }\end{array}$} & Muito Baixas & $-0,87(0,00)$ & $-1,22(0,00)$ & $2,59(99,04) *$ \\
\hline & Baixas & $-1,43(0,00)$ & $-0,48(0,00)$ & $2,11(96,54)^{*}$ \\
\hline & Cerca de $50 \%$ & $-0,67(0,00)$ & $0,54(41,13)$ & $-0,08(0,00)$ \\
\hline & Altas & $0,27(21,29)$ & $-0,18(0,00)$ & $-0,02(0,00)$ \\
\hline & Muito Altas & $0,71(52,32) * *$ & $0,14(11,42)$ & $-0,92(0,00)$ \\
\hline
\end{tabular}

Nota: *Probabilidades fortemente significativas, pois $\mu \times 100 \geq 70 \%$.

${ }^{* *}$ Probabilidades moderadamente significativas, pois $50 \leq \nu \times 100<70 \%$.

Percebeu-se que adolescentes com niveis moderados de estresse foram classificados com alta desesperança e adolescentes com alto estresse foram classificados com baixa e moderada desesperança.

Os dados revelam que $\mathrm{o}$ alto estresse foi associado a adolescentes do sexo feminino. Já o baixo estresse teve associação direta com adolescentes do sexo masculino. Os adolescentes com níveis moderados de estresse também apresentam niveis altos de desesperança; e adolescentes com alto estresse foram classificados com baixa e moderada desesperança.

Os adolescentes com altas expectativas de concluir o ensino médio foram classificados com baixo estresse a partir do Índice ESA. Adolescentes com cerca de $50 \%$ de expectativas de concluir o ensino médio foram classificados com moderado estresse. Adolescentes com baixas e muito baixas expectativas de concluir o ensino médio foram classificados com alto estresse. 
Os adolescentes com expectativas muito altas de ser respeitado foram classificados com baixo estresse. Já adolescentes com baixas ou muito baixas expectativas de ser respeitado foram classificados com alto estresse a partir do índice ESA. Nas demais variáveis das expectativas de futuro ou sociodemográficas, relacionadas aos índices ESA e BHS, não foram encontradas correlações estatisticamente significativas.

Os resultados da análise de correspondência sugerem que o estresse e a desesperança são variáveis que estão relacionadas com as expectativas de futuro mais curtas. Foi verificado que adolescentes com índice de estresse alto e moderado bem como alta desesperança estão associados a adolescentes com baixas ou muito baixas expectativas de concluir o ensino médio, que reflete uma expectativa de curto prazo.

\section{Discussão}

Este estudo apresenta uma análise entre as variáveis estresse, desesperança e expectativas de futuro que é escassa e que apresentou dados relevantes devido às técnicas estatísticas utilizadas. Embora a literatura disponivel apresente essa escassez quanto a estudos que estabeleçam a relação entre essas variáveis juntas na adolescência, as pesquisas que tratam esta temática auxiliam na compreensão dos dados obtidos neste trabalho, mesmo relacionando-as com outras variáveis que não as aqui estudadas.

Os adolescentes investigados, em sua maioria, apresentaram tendências positivas de desenvolvimento, especialmente no tocante às expectativas de futuro quando relacionadas ao nivel de desesperança. Isso vem ao encontro das contribuições colocadas por Hoffman et al. (1995) e o estudo de Oliveira-Monteiro et al. (2012) que defendem a ideia de que os mitos negativos que se formam a respeito da adolescência, não refletem a maioria dos individuos que se encontram nessa fase da vida.

Os dados encontrados indicaram que adolescentes com moderado estresse também apresentaram alta desesperança (baixa esperança), e que adolescentes classificados com alto estresse foram classificados com baixa e moderada de- sesperança (alta esperança). Ao comparar este dado com aqueles obtidos por Yarcherski et al. (2011), observa-se que há uma discordância entre as informações, já que esses autores identificaram relação inversa entre estresse e esperança, ou seja, os maiores escores de estresse foram significativamente associados com menores escores de esperança $(r=-.55)$. Diante dessa relação vale ressaltar que não é possivel pensar em uma inversão linear entre estresse e esperança uma vez que adolescentes com niveis altos de estresse apresentaram nos dados, ainda assim, bons níveis de esperança, ou seja, o estresse nesses adolescentes pode ter assumido o papel de gatilho positivo para a esperança.

Considerando as probabilidades fortemente significativas da correlação entre IESA e IBHS, expectativas de futuro e caracteristicas sociodemográficas dos adolescentes, é possivel afirmar que as meninas são mais propensas ao estresse do que os meninos que foram classificados com baixo estresse. Estes dados são compatíveis com a maioria dos estudos sobre estresse que indicam a tendência das meninas de apresentarem maiores escores de estresse quando comparados com os escores dos meninos (Schraml et al., 2011; Landstedt \& Gadin, 2012; Liu \& Lu, 2012). A revisão sistemática de Marques et al. (2015) confirma que o sexo feminino tem relação positiva com o estresse em populações diferentes e culturas diferentes.

Caires e Silva (2011) verificaram em um estudo com 341 estudantes do $12 .^{\circ}$ ano de escolas secundárias, em Portugal, que o estresse era mais elevado em moças do que em rapazes. Também notaram que o estresse era mais elevado a partir de estressores relacionados à área acadêmica e vocacional $(+=8,288, \mathrm{~g} \cdot \mathrm{l}=194,77 ; \mathrm{p}<0,001) \mathrm{em}$ ambos os gêneros (Mmoças = 2,78; Mrapazes = 2,31 ). As autoras sugerem que quanto maiores as dificuldades vivenciadas na área acadêmica, maior o nivel de estresse, o que confirma os achados deste estudo, visto que os adolescentes com alto estresse apresentavam baixas ou muito baixas expectativas no que se refere à conclusão do ensino médio, enquanto o baixo nível de estresse foi associado com alta expectativa em relação à finalização 
do ensino médio. Esses achados também vão ao encontro dos resultados obtidos na investigação feita por Carmona-Halty et al. (2018), que em uma pesquisa com adolescentes chilenos com idades entre 14 e 17 anos, alunos do ensino médio, verificaram que as emoções positivas, como otimismo, eficácia e esperança, estavam relacionadas com o melhor desempenho acadêmico.

Esses dados confirmam, também, o conceito desenvolvido por Nascimento (2013), que em seu estudo sobre "Projetos de vida" diz que os adolescentes se organizam a partir de dimensões socioeducativa, afetiva, social, e educacional e que essas dimensões são responsáveis pela construção de representações sociais sobre o futuro. Ao verificar que alunos estressados apresentam baixas expectativas de finalizar a etapa atual de sua escolarização, pode haver indicativos de que o estresse interfira diretamente na projeção que o adolescente faz do seu futuro, deixando-o desesperançoso quanto à representação desse futuro na sua realização mais imediata.

Outro dado percebido, dentre as expectativas de futuro pesquisadas, foi a relação entre a expectativa de ser respeitado na comunidade com o estresse, sugerindo que adolescentes com alto estresse têm muito baixas ou baixas expectativas de serem respeitados dentro da sua comunidade, ou vice-versa. Essa expectativa está diretamente ligada à percepção positiva das qualidades do adolescente e constitui uma questão importante quando se está analisando dados obtidos em um país onde há poucas políticas públicas de apoio à juventude como é o Brasil (Brasil, 2010).

O estudo de Oliveira-Monteiro et al. (2011) verificou em uma população de 50 adolescentes estudantes que a maioria deles tinha uma percepção positiva de si mesmo, o que apontou para a positividade no desenvolvimento daqueles adolescentes. Este achado confirmou a ideia de que a imagem mais positiva que os jovens parecem ter de si mesmos pode funcionar como uma defesa perante a negatividade presente na visão que a sociedade adulta tem sobre a juventude, dando-lhes competência social e contribuindo para o seu desenvolvimento positivo.

\section{Considerações finais}

A principal contribuição desse estudo é que os dados apresentados revelaram uma adolescência marcada não apenas pelo pessimismo e desesperança, mas por aspectos positivos, contribuindo para o rompimento da associação entre adolescência e crise ou turbulência.

Notou-se, que estresse e desesperança são variáveis relacionadas com as expectativas de futuro mais curtas, já que nesse item foram associados o estresse alto e moderado de adolescentes com baixas ou muito baixas expectativas de concluir o ensino médio. Pode-se perceber, com base nos resultados, que as meninas estão mais propensas ao estresse eà desesperança do que os meninos o que pode indicar a necessidade de serviços direcionados especificamente para este grupo social com vistasa auxiliar neste processo de transição para a vida adulta.

Refletindo sobre as expectativas do adolescente e suas necessidades, também é possivel afirmar que a valorização e a imagem positiva de si mesmo podem estar relacionadas com as expectativas e o estresse na adolescência e podem comprometer o bem-estar do adolescente. Neste sentido, os dados aqui relatados ressaltam a necessidade de os profissionais da saúde atuarem com vistas a promover a saúde mental nesta população por meio da construção da autoimagem positiva dos adolescentes.

Este trabalho apresenta uma importante limitação que precisa ser considerada, uma vez que a população investigada representa apenas um grupo específico, cuja coleta de dados foi realizada em um único momento no tempo. Neste sentido, sugere-se que pesquisas futuras adotem grupos distintos de adolescentes e assumam uma abordagem longitudinal com vistas a obter dados que melhor revelem o estresse, a desesperança e a sua relação com as expectativas de futuro e as características sociodemográficas dos adolescentes em mais de um momento em suas vidas. Neste sentido, sugere-se estender o estudo a populações diferentes tais como adolescentes em situação de risco, adolescentes de outras regiões do Pará ou do Brasil, adolescentes institucionalizados, não escolarizados, ou mesmo realizar estudos comparativos. 


\section{Referências}

Beck, A. T., Rush, A. J., Shaw, B. F., \& Emery, G. (1997). Terapia cognitiva da depressão (Traduzido por S. Costa). Artmed.

Bock, A. M. B. (2004). A perspectiva sócio-histórica de Leontiev e a critica à naturalização da formação do ser humano: a adolescência em questão. Cadernos Cedes, 24(62), 26-43.

Brasil. (2010). Secretaria de Atenção em Saúde. Diretrizes nacionais para a atenção integral à saúde de adolescentes e jovens na promoção, proteção e recuperação da saúde. Ministério da Saúde.

Bundick, M., Andrews, M., Jones, A., Mariano, J. M., Bronk, K. C., \& Damon, W. (2006). Revised youth purpose survey. Unpublished instrument, Stanford Center on Adolescence, Stanford, CA.

Burrow, A. L., O'Dell, A. C., \& Hill, P. L. (2010). Profiles of a developmental asset: Youth purpose as a context for hope and well-being. Journal of Youth and Adolescence, 39(11), 1265-1273. https://doi.org10.1007/ s10964-009-9481-1

Bussab, W. de. O., \& Morettin. (2002). Estatistica básica (5. ed.). Saraiva.

Caires, S., \& Silva, C. (2011). Fatores de stresse e estratégias de coping entre adolescents no $12^{\circ}$ ano de escolaridade. Estudos de Psicologia, 28(3), 295-306. http://hdl.handle.net/1822/16153

Callina, K. S., Johnson, S. K., Buckingham, M. H., \& Lerner, R. M. (2014). Hope in context: Developmental profiles of trust, hopeful future expectations, and civic engagement across adolescence. Journal of youth and adolescence, 43(6), 869-883. https://doi.org/10.1007/ s10964-014-0096-9

Caspi, A., Houts, R., Belsky, D., Goldman-Mellor, S.,Harrington, H., Israel, S., Meier, M., Ramrakha, S., Shaley, I., Poulton, R., \& Moffitt, T. (2014). The p factor: One general psychopathology fator in the structure of psychiatric disorders? Clinical Psychological Science, 2(2), 119-137. https://doi.org/0.1177/2167702613497473

Carmona-Halty, M., Salanova, M., Llorens, S. J Happiness Stud. (2018). How Psychological Capital Mediates Between Study-Related Positive Emotions and Academic Performance. J Happiness Stud, 20, 1-13. https://doi. org/10.1007/s10902-018-9963-5

Chou, P. C., Chao, Y. M. Y., Yang, H. J., Yeh, G. L., \& Lee, T. S. (2011). Relationships between stress, coping and depressive symptoms among overseas university preparatory Chinese students: a cross-sectional study. BMC public health, 11(1). https://doi.org/10.1186/1471-2458-11-352

Cunha, J. A. (2011). Escalas Beck (1. ed.). Casa do Psicólogo.

Dell'Aglio, D. D., Koller, S. H., Cerqueira-Santos, E., \& Colaço, V. (2011). Revisando o Questionário da Juventude Brasileira: uma nova proposta. In D. D. Dell'Aglio \& S. H. Koller (Eds.), Adolescência e juventude: vulnerabilidade e contextos de proteção (pp. 269-270). Casa do Psicólogo.
Fávero, L. P. L., Belfiore, P.P., Chan, B.L., Silva, F.L. (2009). Análise de dados. Modelagem multivariada para tomada de decisões. Campus.

Folkman, S. (2010). Stress, coping, and hope. Psycho-Oncology, 19, 901-908. https://doi.org/10.1002/pon.1836

Hoffman, L., Paris, S., Hall, E. (1995). Psicología del desarrollo hoy. McGraw-Hill.

Instituto Brasileiro de Geografia e Estatística (2010). Tabelas - Sinopse (Tab 1_12). Censo Demográfico. https:// www.ibge.gov.br/estatisticas/sociais/populacao/ 2098-np-censo-demografico/9662-censo-demografico-2010.html? =\&t=resultados.

Khan, A. (2013). Predictors of positive psychological strengths and subjective well-being among north Indian adolescents: Role of mentoring and educational encouragement. Social indicators research, 114(3), 1285-1293.

Landstedt, E., \& Gådin, K. G. (2012). Seventeen and stressed: Do gender and class matter? Health Sociology Review, 21(1), 82-98. https://doi.org/10.5172/ hesr.2012.21.1.82

Langaro, F. N., \& Benetti, S. P. da C. (2014). Subjetividade contemporânea: narcisismo e estados afetivos em um grupo de adultos jovens. Psicologia Clínica, 26(2), 197-215. http://www w.scielo.br/scielo.php? script=sci_arttext\&pid=S0103-56652014000200012\&lng=en\&tlng=pt

Liu, Y., \& Lu, Z. (2012). Chinese high school students' academic stress and depressive symptoms: Gender and school climate as moderators. Stress and Health, 28, 340-346. https://doi.org/10.1002/smi.2418

Marques, C. P., Gasparotto, G. da S., \& Coelho, R. W. (2015). Fatores relacionados ao nivel de estresse em adolescentes estudantes: Uma revisão sistemática. SALUSVITA, 34(1), 99-108. http://Www.usc.br/biblioteca/ salusvita/salusvita_v34_n1_2015_art_07.pdf

Nascimento, I.P. (2013, maio/ago.). Educação e Projeto de vida de adolescentes do ensino médio [Education and Life Project of high school adolescents]. EccoS Revista Científica, (31), p. 83-100. https://doi.org/10.5585/EccoS.n31.4328

Oliveira-Monteiro, N. R. D., Aznar-Farias, M., Nava, C. D. A., Nascimento, J. O. G., Montesano, F. T., \& Spadari-Bratfisch, R. C. (2012). Estresse, competência e problemas psicológicos de adolescentes estudantes. Arquivos Brasileiros de Ciências da Saúde, 37(1), 23-29.

Patias, N. D.; Machado, W. de L.; Bandeira, D. R.; Dell'Aglio, D. D. (2016). Depression a anxienty and stress scale (DASS-21) - Short Form; Adaptação e validação para adolescentes brasileiros. Psico-ESF, 21(3), 459469. http://dx.doi.org/10.1590/1413-82712016210302

Ramos, E. M. L. S, Almeida, S. S., \& Araújo, A. R. (Orgs.) (2008). Segurança pública: Uma abordagem estatistica e computacional. Editora Universitária EDUFPA.

Rocha, T. B. M., Zeni, C. P., Caetano, S. C., \& Kieling, C. (2013). Mood disorders in childhood and adolescence. Revista Brasileira de Psiquiatria, 35(Suppl. 1), S22-S31. https://dx.doi.org/10.1590/1516-4446-2013-S106

Santrock, J. W. (2014). Adolescência (14. ed.). AMGH Editora Ltda.; Artmed. 
Schermann, L. B., Béria, J. U., Jacob, M. H. V. M., Arossi, G., Benchaya, M. C., Bisch, N. K., \& Rieth, S. (2014). Estresse em adolescentes: estudo com escolares de uma cidade do sul do Brasil. Aletheia, (43-44), 160-173. http://pepsic. bvsalud.org/scielo.php?script=sci_arttext\&pid $=$ S141303942014000100012\&lng $=p t \& t \operatorname{lng}=p t$

Schraml, K., Perski, A., Grossi, G., \& Simonsson-Sarnecki, M. (2011). Stress symptoms among adolescents: The role of subjective psychosocial conditions, lifestyle, and self-esteem. Journal of adolescence, 34(5), 987996. https://doi.org/10.1016/j.adolescence.2010.11.010

Snyder, C. R. (1995). Conceptualizing, measuring, and nurturing hope. Journal of Counseling and Development, 73(1), 355-36o. https://doi.org/10.1002/j.1556-6676.1995.tb01764.x

Snyder, C. R., Harris, C. R., Anderson, J. R., Holleran, S. A., Irving, L. M., Sigmon, S. T., Yoshinobu, L., Gibb, J., Langelle, C., \& Harney, P. (1991). The will and the ways: developmental and validation of an individual-differences measure of hope. Journal of Personality and Social Psycholoy, 60, 570-585. https://doi.or$\mathrm{g} / 10.1037 \% 2$ FoO22-3514.60.4.570

Steinberg, L., \& Morris, A. S. (2001). Adolescent development. Journal of Cognitive Education and Psychology, 2(1), 55-87. http://dx.doi.org/10.1891/194589501787383444

Thapar, A., Collishaw, S., Pine, D., \& Thapar, A. K. (2012). Depression in adolescence. Lancet, 379, 1056-1067. https://doi.org/10.1016/S0140-6736(11)60871-4

Tricoli, V. A. C., \& Lipp, M. E. N. (2011). Escala de Estresse para Adolescentes - ESA. Casa do Psicólogo.

World Health Organization. (2012). Adolescent mental health: Mapping actions of nongovernmental organizations and other international development organizations. Word Health Organization. http://www.who.int/mental_health/ publications/adolescent_mental_health/en/index.html

Yarcheski, A., Mahon, N. E., \& Yarcheski, T. J. (2011). Stress, hope, and loneliness in young adolescents. Psychological Reports, 108(3), 919-922. https://doi. org/10.2466/02.07.09.PR0.108.3.919-922

\section{Cybelle Bezerra Sousa Florêncio}

Mestre em Teoria e Pesquisa do Comportamento pela Universidade Federal do Pará (UFPA), Núcleo de Teoria e Pesquisa do Comportamento (NTPC), em Belém, PA, Brasil; gestora escolar e consultora educacional no Rio Grande do Norte e Paraíba.

\section{Edson Marcos Leal Soares Ramos}

Doutor em Engenharia de Produção pela Universidade Federal de Santa Catarina (UFSC), em Florianópolis, SC, Brasil; professor titular da Universidade Federal do Pará.

\section{Simone Souza da Costa Silva}

Doutora em Psicologia pela Universidade de Brasília (UnB), em Brasilia, DF. Pós doutora em Ciência da Reabilitação pela Universidade de Dortmund, Alemanha; professora e diretora adjunta do Núcleo de Teoria e Pesquisa do Comportamento (NTPC) da Universidade Federal do Pará (UFPA), em Belém, PA, Brasil

\section{Endereço para correspondência}

Cybelle Bezerra Sousa Florêncio

Rua Raimundo Bastos da Silva, 3606, Torre B, apto 1001 Candelária, 59064-610

Natal, RN, Brasil

Os textos deste artigo foram revisados pela Poá Comunicação e submetidos para validação do(s) autor(es) antes da publicação. 